\title{
Brachial Plexus Injuries in Adults: Management and Repair Strategies in our Experience. Results from the Analysis of 428 Supraclavicular Palsies
}

Debora Garozzo $^{1 *}$, Elisabetta Basso ${ }^{1}$, Roberto Gasparotti ${ }^{2}$, Piero Di Pasquale ${ }^{3}$, Fabrizio Lucchin ${ }^{3}$ and Stefano Ferraresi ${ }^{1}$

${ }^{1}$ Department of Neurosurgery, Santa Maria della Misericordia Hospital, Rovigo, Italy

${ }^{2}$ Department of Neuroradiology, University of Brescia, Spedali Civili, Brescia, Italy

${ }^{3}$ Department of Anesthesiology and Intensive Care Unit, Santa Maria della Misericordia Hospital, Rovigo, Italy

\begin{abstract}
Background: Brachial plexus palsies are often devastating injuries leading to severe functional impairment of the upper limb. Their surgical management has greatly changed in the last 30 years and it is still evolving but nowadays the key role of surgery in reversing the destiny of many of these patients is clearly recognized.The Authors are presenting their guidelines of management and repair strategy in the surgical treatment of these injuries.
\end{abstract}

Method: 578 post-traumatic brachial plexus injuries in adults were operated in 23 years: 428 supraclavicular and 150 infraclavicular lesions. A retrospective analysis of the surgical strategies applied in supraclavicular injuries and their outcome has been performed. A follow up of at least two years is available in 303 patients.

Results: In upper brachial plexus injuries, surgery restored good shoulder and elbow function in more than $90 \%$ of cases, regardless the presence of avulsions. Total palsies still retaining some hand function (due to one lower root in continuity with the spine) also received remarkable benefit in about $60 \%$ of cases. Shoulder and elbow function were recovered in $40 \%$ of complete injuries with multiple avulsions.

Conclusions: Advances in preoperative imaging techniques, electrodiagnostic studies and reconstructive surgery have made possible to provide remarkable functional improvement in brachial plexus injuries. Early diagnostic assessment, correct timing of surgery and appropriate rehabilitation are crucial to obtain a rewarding outcome.

Keywords: Supraclavicular brachial plexus injuries; Magnetic resonance myelography; Root avulsions; Spinal accessory nerve transfer; Intercostal nerve transfer; Contralateral C7 transfer

\section{Background}

Although the first description of a brachial plexus (BP) palsy was given by Homer in the duel between Hector and Teucrus in the $8^{\text {th }}$ book of the Iliad, these injuries were recognized as a separate clinical entity among peripheral nerve lesions only in the first half of the $19^{\text {th }}$ century and their surgical repair was first attempted towards the end of the century. During the first decades of the $20^{\text {th }}$ century, the knowledge of these lesions progressed, yet the surgical outcome was so inconsistent and poor that opinion in favor of conservative treatment or amputation of the injured limb grew in the medical community: therapeutic nihilism reached its climax during the SICOT (International Society for Orthopedic Surgery and Traumatology) congress held in Paris in 1966 when a consensus was reached in stating that BP repair should be considered almost impossible and therefore highly discouraged. BP surgery saw the dawn of a new era in the 70's thanks to the pioneering work of Millesi in Vienna and Narakas in Lausanne and since then progress in surgical techniques has been continuous, leading to dramatic improvement in the outcome.

Although in recent years these injuries are progressively gaining more attention, skepticism associated with little knowledge are still harboring in some pockets of the medical community: late referral and early inappropriate management are still a major problem. The price the victims of these devastating injuries pay is high: early diagnostic assessment and correct timing of surgery are crucial to obtain a rewarding outcome. Significant delay can jeopardize the quality of postsurgical recovery especially in avulsive injuries.

This report aims to present the guidelines of management and repair strategy in the surgical treatment of brachial plexus injuries (BPI) according to the Authors' experience, by analyzing a surgical series of 428 supraclavicular lesions.

\section{Methods}

From April 1990 to June 2013 the Authors have operated 578 posttraumatic BPI in adults: 428 supraclavicular and 150 infraclavicular injuries.

Supraclavicular lesions have been retrospectively reviewed, focusing on the diagnostic assessment, the timing of surgery, the surgical findings, the repair strategies and their outcome.

Infraclavicular injuries have been excluded from this study due to their higher variability in findings, with consequent lack of homogeneity in outcome.

\section{Preoperative assessment}

Patients were evaluated clinically (Figure 1) and by neuroradiological (MyeloCT or MR Myelography) (Figure 2) and electrodiagnostic studies (EDS).

Imaging studies are preferably indicated after 3-4 weeks from the causative event due to the fact that pseudomeningoceles need a few weeks to form $[1,2]$.

*Corresponding author: Debora Garozzo, Department of Neurosurgery, Santa Maria della Misericordia Hospital, ULSS 18 Rovigo, Italy, E-mail: debora.garozzo@sem-brachialplexus.com

Received August 17, 2013; Accepted November 26, 2013; Published December 04, 2013

Citation: Garozzo D, Basso E, Gasparotti R, Pasquale PD, Lucchin F, et al (2013) Brachial Plexus Injuries in Adults: Management and Repair Strategies in our Experience. Results from the Analysis of 428 Supraclavicular Palsies. J Neurol Neurophysiol 5: 170. doi:10.4172/2155-9562.1000180

Copyright: @ 2013 Garozzo D, et al. This is an open-access article distributed under the terms of the Creative Commons Attribution License, which permits unrestricted use, distribution, and reproduction in any medium, provided the original author and source are credited. 
Citation: Garozzo D, Basso E, Gasparotti R, Pasquale PD, Lucchin F, et al. (2013) Brachial Plexus Injuries in Adults: Management and Repair Strategies in our Experience. Results from the Analysis of 428 Supraclavicular Palsies. J Neurol Neurophysiol 5: 170. doi:10.4172/21559562.1000180

EDS are also performed after one month as early electromyography (EMG) cannot distinguish between neuroapraxia and other more severe degrees of nerve damage.

After 1994 MRI has almost completely replaced CT myelography, becoming our first choice examination. The MR imaging protocol includes 3 dimensional MR mielography (3DMRM) to detect preganglionic injuries and $\mathrm{MR}$ neurography (MRN) to evaluate postganglionic lesions; Diffusion Tensor Tractography (DTI) has been introduced more recently and, in spite of being an experimental technique that still requires further testing, has already demonstrated high diagnostic accuracy in the assessment of root avulsions [3].

In the last decade, the introduction of phased array coil technology and the advent of parallel imaging with multielement RF-coils have increased the SNR (signal to noise ratio) of high resolution MRI while dramatically shortening imaging times, therefore enabling

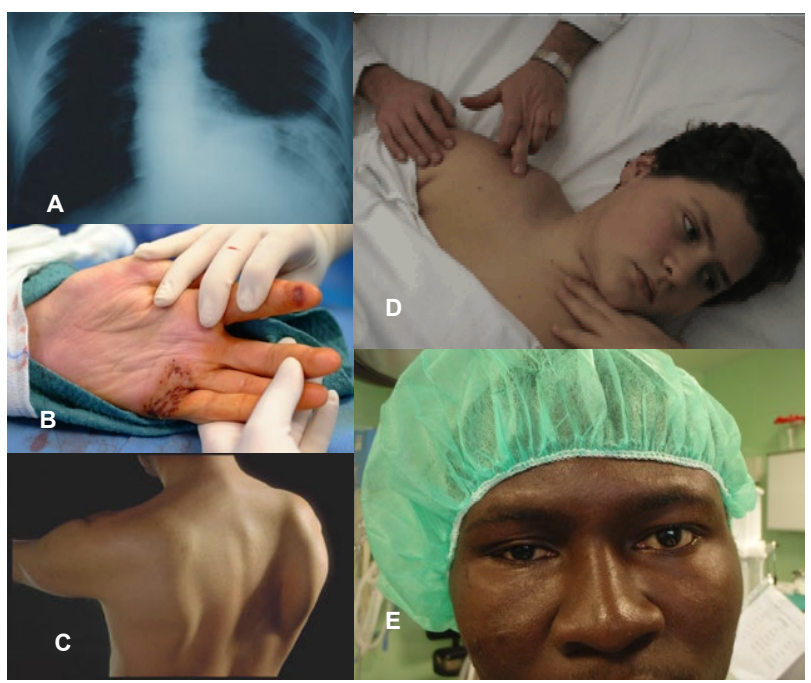

Figure 1: Signs indicating root avulsions (A) phrenic nerve palsy (B) Skin alterations associated with deaferentation pain. (C) winging scapula (D) external pseudomeningocele. (E) Horner's sign.

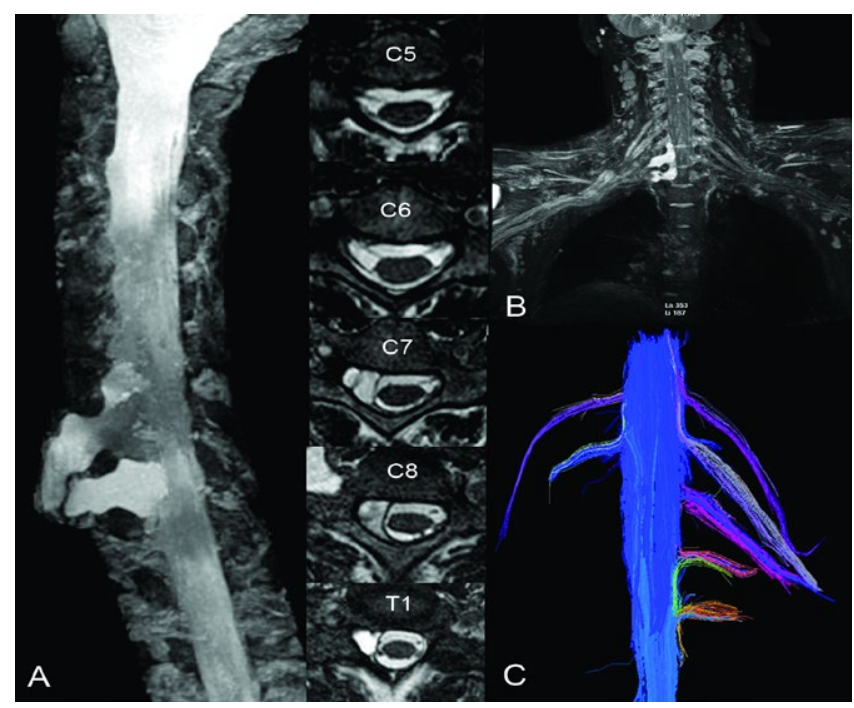

Figure 2: $M R$ myelography (A), neurography (B) and diffusion tensor tractography demonstrating avulsions of $\mathrm{C} 7, \mathrm{C} 8$ and $\mathrm{T} 1$. volumetric acquisition of spine MRI data with multiplanar or curvedplanar reformations. By acquiring high resolution $3 \mathrm{D}$ volumes and subsequently generating multiplanar reformats or even curved-planar reformats, MR imaging has become more efficient, following the same trend introduced by Multidetector CT (MDCT). Improved SNR at 1.5 $\mathrm{T}$ and decreased cerebrospinal fluid (CSF) pulsation artifact as well as increased spatial and contrast resolution enhance the sensitivity for identifying cervical nerve roots.

At present the Authors perform MR investigation of BPI on $1.5 \mathrm{~T}$ scanners (Aera, Avanto, Siemens, Erlangen, Germany) with a standard 8-channel transmit-receive neck coil, used in combination with a thoracic coil in a phased array.

MRM is based on a sagittal 3D T2 SPACE sequence (sampling perfection with application optimized contrasts using different flip angle evolution, repetition time [TR]/echo time [TE], 1500/118 milliseconds; isotropic voxel, $0.8 \mathrm{~mm}$; field of view [FOV], $250 \mathrm{~mm}$;). The 3D T2 SPACE sequences are currently preferred to the standard 3D CISS (constructive interference in steady state) sequences for MRM studies because they are insensitive to the artifacts caused by CSF pulsatile motion. The 3D MRM dataset is then processed with a MIP (maximum intensity projection) algorithm to obtain myelogramlike images. As MIP artifacts can cause inconstant visualization of nerve roots within the dural sleeves on MIP views, 0.7-1 mm-thick reformatted axial oblique sections are obtained from the same data set with the multiplanar reconstruction (MPR) algorithm. The axial reformatted sections are oriented with a progressive increase of the angle with the intervertebral disk from $\mathrm{C} 4$ to $\mathrm{T} 2$ following the direction of nerve roots, which changes from $\mathrm{C} 5$ to $\mathrm{T} 1$. The reformatted paraxial sections are simultaneously rotated in 2 different planes to best depict their attachment to the spinal cord. The nerve rootlets must be identified on several sections and compared with those on the opposite side to avoid false positive findings from abnormal posture caused by shoulder trauma. The whole spectra of imaging findings, including traumatic pseudo-meningoceles and partial or complete root avulsions can be easily identified with carefully selected submillimetric multiplanar reformats.

DTI is a cutting-edge technique for studying peripheral nerves, which cannot be as readily and reliably performed in the spinal cord and brachial plexus for technical and anatomical reasons such as susceptibility and pulsation artifacts as well as motion of organs in the thorax. The DTI acquisition consists of an axial single-shot spin echo planar imaging sequence (TR/TE, 9200/90 milliseconds; matrix, $86 \times$ 114; FOV, $251 \mathrm{~mm}$; isotropic voxel, $2.2 \mathrm{~mm}$; contiguous axial sections from C3 to T2, 72; NEX5 b value, $900 \mathrm{~s} / \mathrm{mm}^{2}$; gradient directions, 12; parallel imaging and total scan time, 11 minutes and 40 seconds). Respiratory triggering is also applied to minimize motion.

Using the DTI software installed on the workstations provided by the MR scanners manufacturers, root avulsions can be defined on MR tractography as a loss of continuity with the spinal cord fibers.

The MRN assessment of postganglionic injuries is based on a coronal oblique 3D T2 short time inversion recovery SPACE sequence (TR/time inversion [TI]/TE, 2000/180/459 milliseconds; slice thickness, $1 \mathrm{~mm}$; FOV, $250 \mathrm{~mm}$ ). This technique provides enhanced contrast between nerves and muscles and offers the extra advantage of generating oblique and curved-planar reformations of nerve roots, primary trunks and cords. Traction injuries of the BP are characterized by increased signal intensity and size and can be easily distinguished from the adjacent vessels and surrounding muscles. 
Citation: Garozzo D, Basso E, Gasparotti R, Pasquale PD, Lucchin F, et al. (2013) Brachial Plexus Injuries in Adults: Management and Repair Strategies in our Experience. Results from the Analysis of 428 Supraclavicular Palsies. J Neurol Neurophysiol 5: 170. doi:10.4172/21559562.1000180

\section{Timing of surgical treatment}

Patients were referred from all over the Country and the referral time was very variable: it ranged between 1 and 13 months, yet most cases were referred after 4-5 months from the traumatic event.

Primary nerve repair was always performed between 2 months and 1.5 years from the injury even if the timing of surgery was obviously depending on the referral time: in the majority of cases, patients were operated after 5-6 months from the injury.

For the early referrals, the Authors advised surgery as soon as possible once avulsions were revealed at MRI, within 2-3 months from the injury. In postganglionic injuries, indication to surgery was given when 5-6 months after the injury, no spontaneous recovery was detected clinically and/or at EMG.

In less than $20 \%$ of complete palsies, after a few weeks from the trauma a partial recovery of the hand function occurred, due to a lower root in continuity with the spine. In these injuries ("total palsy with one-root hand"), the viable time window was also extended to 6-8 months, hoping to restore a M3 hand that would allow the option of the infraclavicular nerve transfer that will be described below.

\section{Procedural approach and surgical technique}

Surgery is performed in the supine position under general anesthesia, no muscular blocks are used. It usually includes two stages: exploration of the brachial plexus and its microreconstruction via an anterior approach. Exploration is not performed in lower plexus injuries, root avulsions having always been clearly revealed at MRI.

Loupes are used during the exploration; nerve sutures are performed with nylon 9-0 and fibrin glue (Tissuecol/Tisseel by Baxter) under the microscope.

Frozen examinations of the root stumps were used till 1996.

Intraoperative, direct neuroelectrical stimulation is normally used. Nerve action potentials (NAPs) are not used. EDS are always applied for contralateral $\mathrm{C} 7$ (CC7) transfer to minimize the risk of possible complications at the donor site. In such cases we study the innervation dominance of the extensor muscles and biceps in the donor limb. Once the supraclavicular donor plexus is exposed, the three upper trunks and both the divisions of the middle trunk (MT) are selectively stimulated and compound muscle action potential (CMAP) are recorded from the triceps, extensor digitorum communis (EDC), flexor carpi radialis, extensor indicis proprius and biceps.

The AUC (area under curve) of CMAP recorded during stimulation of the MT and its division is compared to the sum of the AUCs of CMAP recorded by stimulating the upper and lower trunks. The MT posterior division is considered an expendable donor if its contribution to the triceps and the EDC innervation is less than 50\% when compared to the sum of the upper and lower trunks contributions. If it is superior to $50 \%$, its use as donor nerve is ruled out (this happened in 3 cases out of 21). When the calculations were doubtful to employ the whole posterior contribution, either the anterior contribution ( 2 cases) or half of the posterior contribution (1 patient) were transected (Figure 3 ).

Regarding the microreconstruction, a homogenous repair strategy is not possible due to the high variability of the surgical findings (Figure 4), especially in the panplexopathies.

It was occasionally necessary to resort to techniques not routinely used (end to side techniques, transfer of motor branches from the cervical plexus, intramuscular neurotization etc.) in order to restore functional priorities under particular circumstances: these cases are not statistically relevant and have been excluded from the description of repair strategies.

Following are our preferred options for microreconstruction in each injury pattern.

\section{Preferred repair strategies in C5, C6 (C7) injuries}

196 patients were submitted to surgery (Figure 5), aiming to restore shoulder function and elbow flexion. Before 1995, a graft reconstruction of the upper trunk (UT) with the accessory nerve (XIN) transfer to the suprascapular nerve (SSN) was the repair strategy in these injuries (9 cases). After 1996, graft reconstruction to regain the biceps was abandoned in favor of the technique popularized by Oberlin [4], performed in 43 cases.

After 1998 microreconstruction for these injuries includes:

(a) A XI - SS nerve transfer;

(b) A thoracodorsal (occasionally using half of the nerve) or subscapular nerve transfer to the axillary nerve (AXN). Reinnervation of the deltoid by a triceps branch of the radial was occasionally attempted via the anterior approach. When such donors aren't available (in C5, C6, C7 injuries), a graft reconstruction from an available root stump to the UT posterior contribution is the alternative option.

(c) A transfer of fascicles from the medial cord to the musculocutaneous nerve (MCN) at infraclavicular level: this is a technical variant of Oberlin's procedure designed by the Authors and its description is following.

The medial cord bifurcation into ulnar nerve and medial root of the median nerve is approached and repeatedly stimulated at low intensity $(0.2$ and $0.3 \mathrm{~mA})$ to identify the more powerful fascicles for the wrist flexors or pronators. Fibers to the intrinsic hand muscles are excluded, for fear of possible complications: therefore the fascicles directed to the ulnar nerve are generally excluded in favor of those to the median nerve. Once the donor fascicles are identified, they are transected and the MCN is sectioned at its origin from the lateral cord and brought directly into apposition with their distal surface. Suture without tension is performed: no relevant surface mismatch is generally found.

\section{Preferred repair strategy in C8-T1 injuries}

7 patients (Figure 6) sustained a lower plexus injury. Before 2009 the

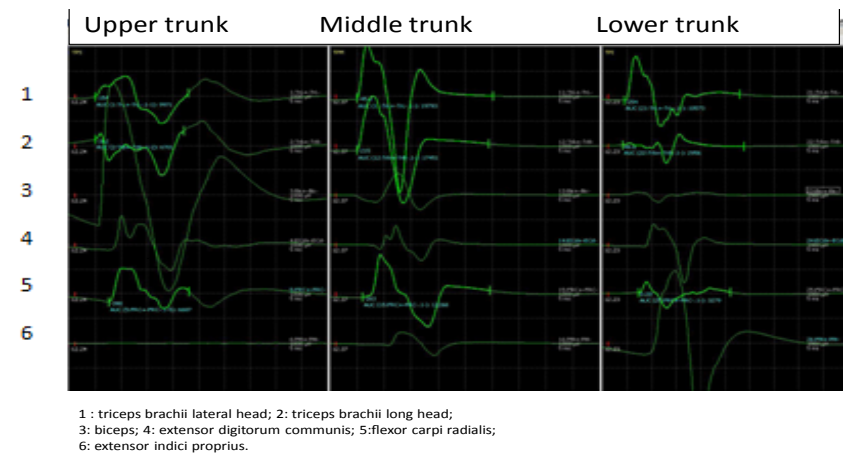

Figure 3: The AUC of CMAP of the lateral (1) and long head (2) of triceps recorded by stimulating the middle trunk are more than $50 \%\left(37.244 \mu \mathrm{V}^{2}\right)$ o the sum of the same AUC recorded by stimulating the upper and lower trunks $\left(30.191 \mu \mathrm{V}^{2}\right)$. Note that flexor carpi radialis (4) has also a major contribution from the middle trunk. In this case the harvest of contralateral C7 has not been performed. 
Citation: Garozzo D, Basso E, Gasparotti R, Pasquale PD, Lucchin F, et al. (2013) Brachial Plexus Injuries in Adults: Management and Repair Strategies in our Experience. Results from the Analysis of 428 Supraclavicular Palsies. J Neurol Neurophysiol 5: 170. doi:10.4172/21559562.1000180

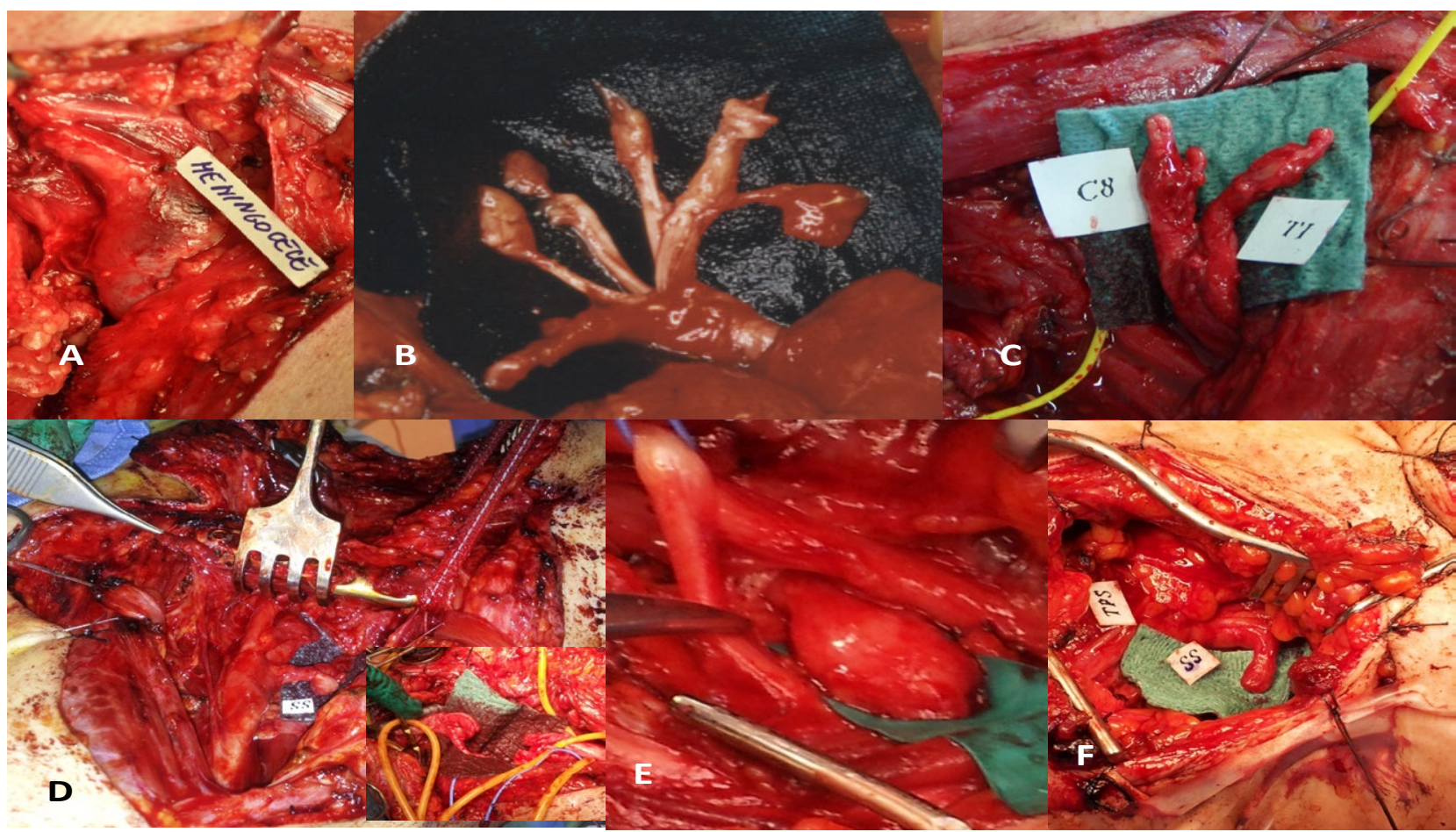

Figure 4: Surgical findings at exploration of the brachial plexus: (A) pseudomeningocele, (B and C) root avulsions and (D) a peculiar case of complete brachial plexus palsy with a clavicular fracture previously repaired elsewhere: during the orthopedic procedure it was already documented that the trunks had been severed by the sharp stumps of the fractured clavicle. At exploration, it was found that the proximal and distal stumps of the severed 3 upper trunks and the supraclavicular nerve were embedded in a mass of scarred and fibrous tissue under the repaired clavicle; the proximal stump of the lower trunk was also adherent to the subclavian artery. The inner square shows the stumps of the upper trunk and its contributions once they were prepared for the graft repair. (E) Supraclavicular injury of the brachial plexus coexisting with rupture of the axillary nerve before its inlet into the quadrangular space: note the bulky neuroma on the proximal nerve stump (between the MC and the lateral root of the median nerve). (F) Rupture of the suprascapular nerve.

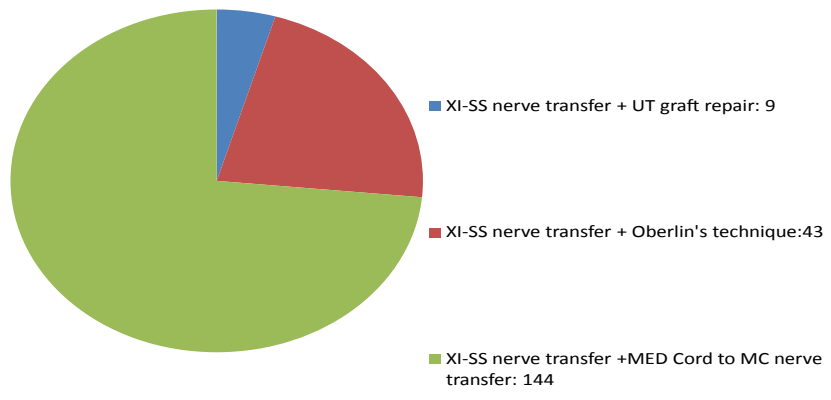

Figure 5: No. of cases operated according to each type of repair strategy in C5, C6 (C7) injuries.

brachialis branch of MCN was transferred to the anterior interosseus nerve (AIN) with a short interposition graft (4 patients).

In the last 3 cases, the donor branch was directly transferred to the fascicles for the finger flexors of the median nerve.

\section{Preferred repair strategy in complete injuries}

In panplexopathies, our surgical priorities were the spinati muscles and the biceps whereas the deltoid and triceps were considered secondary targets. Hand re-animation was attempted only after 2009.

Complete post-traumatic plexopathies accounted for 225 cases (Figure 7).
The spinati muscles were generally restored through a XI-SS nerve transfer.

Concerning the other goals, repair strategies varied according to the findings:

(A) In 125 patients exploration revealed at least one root (generally C5) in continuity with the spinal cord. In the past, reconstructive strategy was often a plexo-plexal repair, bridging the usually found neuroma-in-continuity with grafts from the expendable root stump to both UT divisions (graft length ranging between 4 to $12 \mathrm{~cm}$ ), hoping for successful regeneration that would restore more than one target muscle. Grafts could also be directed to the lateral cord/MCN (graft length ranging from 15 to $20 \mathrm{~cm}$ ).

In recent years, the only available root stump is preferably grafted to the UT anterior division to secure elbow flexion: further targets (mainly the deltoid and pectoral muscles) are included in the reconstructive plan if another plexal donor is revealed, otherwise we may resort to alternative donors (e.g. intercostal nerve transfer to the radial nerve).

In order to supplement the number of target muscles, in the years 1997-1998 in 8 patients sural grafts (length between 20 and $30 \mathrm{~cm}$ ) were coapted to fascicles from the contralateral medial cord, passed under the skin across the chest and used to neurotize the radial or the axillary nerve.

In 10 patients a CC7 transfer was attempted to obtain re-animation of the hand: in two cases to the lower trunk with an interposition graft, 
Citation: Garozzo D, Basso E, Gasparotti R, Pasquale PD, Lucchin F, et al. (2013) Brachial Plexus Injuries in Adults: Management and Repair Strategies in our Experience. Results from the Analysis of 428 Supraclavicular Palsies. J Neurol Neurophysiol 5: 170. doi:10.4172/21559562.1000180

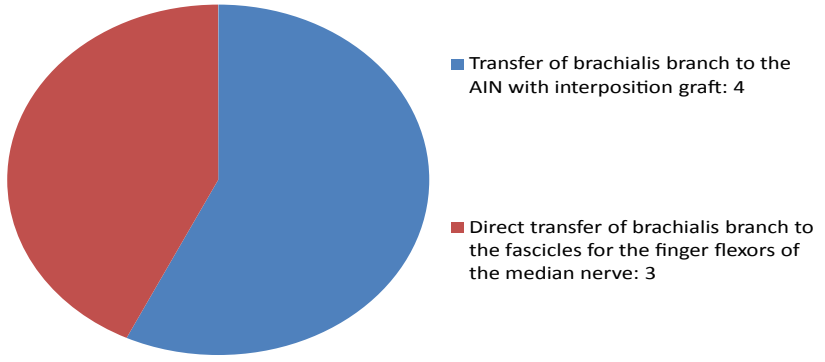

Figure 6: No. of patients operated according to each repair strategy in C8-T1 injuries.

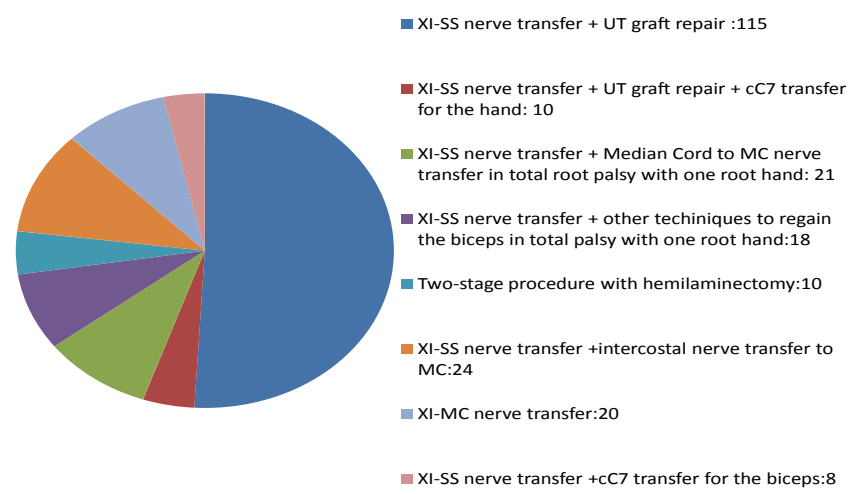

Figure 7: No. of patients operated according to each repair strategy in complete brachial plexus injuries.

in the other cases the recipient nerve was the median nerve according to the two stage-technique described by $\mathrm{Gu}[5]$.

(B) The "total palsy with one root hand" injury occurred in 39 cases. In 18 patients plexo-plexal graft repair, intercostal nerve transfer or phrenic nerve transfer were performed to regain the biceps; after 1998 21 patients (flexors-intrinsic muscles scoring M3-4 in 15 cases and M1$\mathrm{M} 2$ in 6 cases) received the infraclavicular median cord-MCN transfer.

(C) In 61 patients the lesion was either clearly panavulsive or the roots left in apparent continuity were significantly scarred to allow a successful plexo-plexal repair.

During the years 2003 and 2004, 10 cases were operated through the following strategy. Via a hemilaminectomy, sural nerve grafts (length ranging between 15 and $20 \mathrm{~cm}$ ) were coapted to half of the C4 ventral rootlets and passed under the skin of the neck to reach the supraclavicular area; a few days later, their distal ends were coapted to the UT divisions via the usual anterior approach.

In 24 patients the III, IV and V intercostal nerves were transferred to the MCN to restore elbow flexion. From 2012 their sensory branches are neurotized to the lateral root of the median nerve (transected at the lateral cord bifurcation and brought directly into apposition) attempting to restore protective sensation in the hand.

In 26 cases, due to severe thoracic trauma with multiple rib fractures or Brown Sequard's syndrome, intercostal nerve transfer had to be ruled out.

Before 2008, in such patients (20 cases) the only expendable donor (the accessory nerve) was directly transferred to the UT anterior contribution or the lateral cord $/ \mathrm{MCN}$ with an interposition graft (length of graft between 8 and $20 \mathrm{~cm}$ ): restoration of shoulder function had to be given up in favor of elbow flexion, considered the main priority.

After 2009, CC7 was transferred to the UT or its anterior contribution with an interposition graft in 3 cases and in 5 to the MC nerve via the two stage procedure.

\section{Postoperative care}

We maintain the limb in a shoulder immobilizer for 2 weeks and advise the patient to resume physiotherapy after this period.

We do not have a standardized rehabilitation protocol: after the surgery, patients return to their area of origin to be taken in charge by their local therapists that design the rehabilitation treatment according to the specific needs of the patients.

\section{Results from surgery}

In our series patients' age ranged from 14 to 63 years, although $90 \%$ of the injuries occurred between the second and the fourth decades. Only $5 \%$ of patients were women.

The causative mechanism was a motorcycle accident in more than $90 \%$ of cases.

\section{Complications from surgery}

Surgery was generally uneventful with few exceptions:

a) Dyspnea under effort for less than 3 months, due to partial injury of a double phrenic nerve during dissection of tight scar to expose upper root stumps (postoperative RX demonstrated hemidiaphragmatic palsy),

b) An infraclavicular hematoma due to bleeding from a cephalic vein branch and a hematoma in the calf after a sural nerve harvest. Both required surgical evacuation.

c) A hemothorax due to pleural tear during intercostal nerve harvest in a patient under warfarine. It required insertion of a chest tube.

Regarding the harvest of the CC7, one patient presented a wrist drop that recovered almost completely in some months, two patients complained of mild weakness in the triceps for about 20 days, two patients (the MT anterior division employed as donor) presented mild paresthesias for less than two months in the radial territory.

\section{Follow up}

A follow up of at least two years is available for 303 patients. Results of CC7 transfer are available only for direct transfer to the UT: this technique was introduced after 2009 and when performed through the two stage-procedure, it needs at least 3 years of follow up to evaluate its outcome.

Results are considered favorable when muscle strength scores equal or superior to M3 according to the British Medical Council scale [6].

Muscle strength graded M2 has limited functional value but secondary tendon and muscle transfers may supplement the disappointing result of the primary repair. In case of failed graft repair, if an expendable donor can be identified, the fall-back plan is a secondary nerve transfer.

We report only favorable results of primary repair. 
Citation: Garozzo D, Basso E, Gasparotti R, Pasquale PD, Lucchin F, et al. (2013) Brachial Plexus Injuries in Adults: Management and Repair Strategies in our Experience. Results from the Analysis of 428 Supraclavicular Palsies. J Neurol Neurophysiol 5: 170. doi:10.4172/21559562.1000180

The spinati muscles are evaluated measuring the degrees of shoulder abduction (equal or more than $50^{\circ}$ ) and extrarotation (equal or more than $30^{\circ}$ ).

Following are results of microreconstruction for each injury pattern. Overall results for shoulder reinnervation are summarized in Table 1 and results for biceps reinnervation are reported in Table 2.

Upper brachial plexus injuries: follow up data are available for 191 patients. In more than $90 \%$ of these cases, the XI-SS nerve transfer achieved shoulder stability and good abduction, valid extrarotation was restored in about $30 \%$ of the patients. Nerve transfers allowed deltoid recovery in $50 \%$ of cases: the thoradocorsal nerve proved to be an excellent donor but mostly when the whole nerve was transected.

Concerning biceps reinnervation, graft repair gave favorable results in 5 out of 6 patients. For the 43 cases that received Oberlin's technique, results were already published in 2004 [7]. With one exception, the infraclavicular transfer always provided excellent restoration of elbow flexion, allowing reinnervation of both biceps and brachialis muscles.

Lower plexus injuries: Follow up data are available in 4 patients. The direct brachialis branch transfer to the median nerve allowed M4 finger flexors recovery in 2 patients; the transfer to the AIN with an interposition graft failed in both the remaining cases.

Complete injuries of the brachial plexus: 79 patients that had received a XI-SS nerve transfer were available at follow up. In "total palsies with one root-hand", valid shoulder abduction was achieved in $60 \%$ of cases and functional extrarotation occurred in $10 \%$. In flail arms, valid shoulder abduction was restored in $40 \%$ of cases, no patient recovered extrarotation.

Graft repair achieved favorable results in less than $50 \%$ of the 57 patients available at follow up. Functional outcome was strictly depending on the graft length: with the exception of one case (graft length $15 \mathrm{~cm}$ ), no satisfactory outcome occurred with grafts longer than $12 \mathrm{~cm}$. Graft repair from one plexal donor to more than one target mostly proved unsuccessful.

Grafts failed completely in 9 out of the 10 cases where the strategy

\begin{tabular}{|l|l|l|}
\hline \multicolumn{3}{|c|}{ Results of spinati muscles restoration after XI-SS nerve transfer } \\
\hline No. of patients for each injury pattern & Abduction & Extrarotation \\
\hline Upper brachial plexus: 191 & $90 \%$ & $30 \%$ \\
\hline Total palsy with one root- hand: 25 & $60 \%$ & $10 \%$ \\
\hline Complete brachial plexus: 60 & $40 \%$ & $0 \%$ \\
\hline
\end{tabular}

Results for the spinati muscles are considered favorable and here reported when shoulder abduction is equal or more than $50^{\circ}$ and extrarotation is equal or more than $30^{\circ}$

Table 1: Results of shoulder reinnervation in partial and complete injuries of the brachial plexus. implied the hemilaminectomy and the C4 spinal nerve was the donor nerve.

No results were obtained in any of the 8 cases where contralateral medial cord fascicles were neurotized to the axillary or radial nerve.

Concerning the MCN reinnervation:

(a) The XI nerve transfer allowed a favorable result (M3) only in one case out of 14 available at follow up: the donor nerve had been directly transferred to the UT.

(b) Intercostal nerve transfer resulted in functional recovery in $30 \%$ of the 9 patients found at follow up.

(c) The infraclavicular transfer achieved good elbow flexion in $70 \%$ of the 15 cases whose wrist flexors were graded M3-4; in the 4 patients whose wrist flexors scored M1-M2 preoperatively and that were available at follow up, no valid elbow flexion was restored.

(d) Out of the 3 cases available at follow up, CC7 transfer to the UP allowed one patient to recover excellent biceps strength but she has severe difficulties in flexing her elbow independently from the contralateral latissimus dorsi activation (4 years have elapsed from the procedure).

\section{Discussion}

Brachial plexus injuries (BPI) probably occur in $1 \%$ of the patients presenting to a trauma facility [8]. Being traction injuries generally occurring during motorcycle accidents, they mostly affect young males: therefore, regardless of their rarity, these lesions have a relevant social importance, often leading to an impairment or permanent disability of the upper limb in individuals at the very beginning or at the peak of their productive years with severe psychological distress caused by an event that abruptly changed their life forever.

Surgery can undoubtedly reverse the destiny of many of these patients but early diagnostic assessment, correct timing of surgery and an intensive pre and post operative rehabilitation treatment are crucial in order to obtain rewarding results.

The first step of the diagnostic assessment is to reveal the presence of root avulsions: preganglionic injuries have no chances to recover spontaneously.

In our experience [1-3], 3D MRI (conventional MRI of the cervical spine is of no help) represents the core of BPI preoperative assessment.

Unfortunately a world-wide diffusion of MRI to study BPI is unlikely under the present circumstances: MRI involves demanding costs which cannot be afforded in many countries and, in order to become reliable and reproducible, it should be performed by a neuroradiologist with an established experience in the technique.

\begin{tabular}{|c|c|c|c|c|}
\hline \multicolumn{5}{|c|}{ Results of biceps reinnervation according to each surgical technique } \\
\hline & $\begin{array}{l}\text { No. of patients available at } \\
\text { follow up for each injury } \\
\text { pattern and results from graft } \\
\text { repair }\end{array}$ & $\begin{array}{l}\text { No. of patients available at } \\
\text { follow up and results from } \\
\text { intercostal nerve transfer }\end{array}$ & $\begin{array}{l}\text { No. of patients available } \\
\text { at follow up and results } \\
\text { from XI-MC transfer with an } \\
\text { interposition graft }\end{array}$ & $\begin{array}{l}\text { No. of patients available at } \\
\text { follow up for each injury } \\
\text { pattern and results from } \\
\text { infraclavicular transfer }\end{array}$ \\
\hline Upper brachial plexus & 6 pts: $85 \%$ & & & 142 pts: $99 \%$ \\
\hline Total palsy with one root hand & 6 pts: $50 \%$ & & & $\begin{array}{l}15 \mathrm{pts}^{\star}: 70 \% \\
4 \mathrm{pts}^{\wedge}: 0 \%\end{array}$ \\
\hline Complete brachial plexus & 51 pts: $50 \%$ & 9 pts: $30 \%$ & 13 pts: $0 \%$ & \\
\hline
\end{tabular}

Results are considered favorable and here reported when muscle strength scores equal or superior to M3

${ }^{*}$ Patients whose wrist flexors scored M3-M4 preoperatively

^Patients whose wrist flexors scored M1-M2 preoperatively

Table 2: Results of biceps reinnervation in partial and complete injuries of the brachial plexus. 
Citation: Garozzo D, Basso E, Gasparotti R, Pasquale PD, Lucchin F, et al. (2013) Brachial Plexus Injuries in Adults: Management and Repair Strategies in our Experience. Results from the Analysis of 428 Supraclavicular Palsies. J Neurol Neurophysiol 5: 170. doi:10.4172/21559562.1000180

We rely less on EDS as the information given by such techniques is inferred and not directly demonstrated.

After the trauma, patients should start intense physiotherapy as soon as their general conditions allow: if rehabilitation is neglected or inappropriate, musculoskeletal degenerations and joint stiffness rapidly develop, ultimately impairing the outcome. Rehabilitation program should be tailored for each patient focusing on his/her specific needs. Slings and shoulder supporters are advisable to prevent or minimize the development of problems caused by the distractive forces of the weight of the denervated upper limb: wrist and elbow are also at risk but the shoulder is the joint most frequently impaired and, if left unattended, glenohumeral subluxation can become quite pronounced. Pain management is also crucial to help with participation in physiotherapy.

Concerning surgical technique, it must preliminarily stated that in spite of the advances of the imaging, we do believe that exploration has still a role, especially in panplexual injuries, being the only way to completely assess the extent, level and severity of the injury. Moreover in about $5 \%$ of supraclavicular injuries there might also be a distal rupture of the MC or the AX at infraclavicular level that would invariably lead to failure in case of supraclavicular graft repair.

The analysis of our surgical series has to be evaluated at the light of an experience that progressively ruled out unsuccessful techniques: these are the cases of the hypoglossal nerve transfer $[9,10]$, the accessory nerve transfer to the MCN with an interposition graft, the use of donor fascicles from the contralateral medial cord or from the $\mathrm{C} 4$ spinal root via hemilaminectomy.

On the other hand, new strategies allowed to achieve results not expected before: Oberlin's technique is undoubtedly the equivalent of the Copernican revolution in BP surgery and opened the way to a new philosophy of treatment.

The here described infraclavicular transfer for biceps reinnervation was clearly inferred by his technique. We consider this transfer perfectly feasible and it has allowed restoration of a valid elbow flexion not only in $\mathrm{C} 5, \mathrm{C} 6(\mathrm{C} 7)$ palsies but also in the "total palsy with one root hand" injuries (that must be distinguished from upper brachial plexus injuries) when wrist flexors had recovered to grade 3/4. Moreover it provides reinnervation of both biceps and brachialis muscles at the same time, making unnecessary the double transfer designed by Oberlin himself. Since the site of coaptation between donor and recipient is more proximal than in the original technique, this procedure requires slightly longer times to reveal the first signs of recovery ( 7 months) but this does not influence the final quality of the reinnervation.

Direct nerve transfers are undoubtedly the winning strategy in BP repair and should be the technique of first choice whenever possible: in our hands as well as in the literature [11-13], they provide a faster and better recovery compared to graft repair. A favorable outcome is generally guaranteed even when a considerable time has elapsed from the trauma.

When donors are carefully selected, nerve transfers have few downsides but they are not flawless techniques. Moreover it must be remembered that the transection of the donor nerve may imply the loss of function in its original target muscle: the so denervated muscle becomes no longer suitable for secondary tendon/muscles transfers (e.g. the latissimus dorsi after resection of the thoracodorsal nerve to restore deltoid) and this must be kept in mind when planning the primary repair.

Concerning graft reconstructions, experience has demonstrated that successful outcome is strictly depending on the quantity of myelin found in the stump [14] (whereas the donor in the transfer is always a healthy nerve) and the graft length (the best results are possible with short grafts).

In plexo-plexal repair, we have also learned that if only one expendable donor root is available, it is preferable to employ it to restore only one function (elbow flexion) in order to supplement the chances of a favorable outcome.

Concerning the quality of the reinnervation, it is interesting to note that the XI-SS nerve transfer restores shoulder abduction in higher percentage than extrarotation and the general severity of the injury affects the outcome: there is a remarkable loss of functional results in total palsies in comparison to upper palsies, probably not only due to the overall more severe muscle denervation of the upper extremity but also to the cortical reorganization. Bertelli has reported similar results [15].

The severity of the injury also seems to affect the chances of successful graft repair according to the different clinical presentation: grafts gave favorable results in almost $85 \%$ of cases when performed in C5, C6 (C7) palsies whereas they achieved valid functional restoration in $50 \%$ of patients with complete injuries.

Some might notice a limited use of intercostal nerve transfers in our hands: we have a high percentage of severe thoracic trauma (multiple rib fractures) associated to the upper extremity injury and therefore we are often obliged to rule out this option.

We seldom performed a phrenic nerve transfer even if it is widely recognized that this nerve is a good donor. The literature [16] is clear about the adjustment of the respiratory function in about one year after the hemidiaphragmatic palsy has occurred (especially when affecting the left side), yet no reports are available in the long run when age itself starts to affect the respiratory function.

Our patients are all very young and the Italian life expectancy is presently 85 years (the second longest one all over the world) and likely to increase in the next future. Moreover patients often smoke and are obese (many actually become obese because of the stationary lifestyle they are obliged to after the trauma or the onset of a depression which leads them to give up sport and change their alimentary habits).

After 2008 we have also adopted the use of CC7 transfer but our experience is still limited to assess its validity especially concerning the possibility to re-animate the hand. Western surgeons often prefer not to resort to this procedure fearing complications at the donor limb but this occurrence can reasonably be excluded with the application of intraoperative electrodiagnostic testing methods When other options have to be ruled out, CC7 certainly represents the only hope to restore some function in an upper limb that would otherwise be condemned to complete disability. Our main concern is only whether the patient will ultimately be able to autonomize the restored function from the activation of the donor side: for this reason we have never chosen to perform this technique in patients who sustained severe head injury, fearing that their neuroplasticity had been compromised. It must be considered that functional autonomization is likely to require long times: cortical reorganization probably needs more than 5 years [17]. Rehabilitation focused on central re-education can also be crucial in developing the ability to dissociate the movements of the two upper extremities.

Our last remarks concern the limitations of this study. It was a retrospective review of 428 supraclavicular injuries operated in a 
Citation: Garozzo D, Basso E, Gasparotti R, Pasquale PD, Lucchin F, et al. (2013) Brachial Plexus Injuries in Adults: Management and Repair Strategies in our Experience. Results from the Analysis of 428 Supraclavicular Palsies. J Neurol Neurophysiol 5: 170. doi:10.4172/21559562.1000180

Page 8 of 8

period over 23 years: as it was previously pointed out, this necessarily influenced the repair strategy, as the surgeons applied different techniques according to the historical period when they were referred the patients, this variety in the surgical procedures certainly contributing to the non homogeneous results found at the follow up. Another important limitation is given by the very selection of the patients: they were sent to the Authors' observation by other physicians with remarkable differences in the referral time and consequently in the surgical timing, thus reducing the possibility of a rewarding outcome when significant delay occurred, especially in panavulsive injuries. The lack of a standardized rehabilitation protocol is also to be considered when analyzing the final outcome. Yet it must be admitted that up till now, the possibility to perform a prospective study with homogeneous criteria especially as far as the correct timing of surgery is concerned, is rather utopian given the terms of the reality we operate in.

\section{Conclusions}

The last decades have seen a tremendous improvement in the field of brachial plexus surgery, due to the development of preoperative imaging studies, electrodiagnostic testing methods and the introduction of new, ingenious reconstructive techniques.

The outcome of upper brachial plexus injuries has been dramatically improved: nowadays an almost normal upper limb function can be restored even when avulsions are revealed.

In complete injuries retaining a partial function of the hand, a satisfactory improvement can be achieved in a remarkable percentage of cases.

Only in panavulsive injuries, surgery is often only salvage-like in nature and these patients are still condemned to severe disability.

Early referral to the surgeons and correct rehabilitation, tailored to focus the specific needs of the patient, are of paramount importance to improve surgical outcome.

\section{References}

1. Gasparotti R (2012) Radiographic assessment of adult brachial plexus injuries. In: practical management of pediatric and adult brachial plexus palsies. Kevin C Chung, John E McGillicuddy Lynda, Yang J-S (eds.) Elsevier LTD, London, USA, 234-249.

2. Gasparotti R, Ferraresi S, Pinelli L, Crispino M, Pavia M, et al. (1997) Threedimensional MR myelography of traumatic injuries of the brachial plexus. AJNR Am J Neuroradiol 18: 1733-1742.
3. Gasparotti R, Lodoli G, Meoded A, Carletti F Garozzo D, et al (2013) Feasibility of diffusion tensor tractography of brachial plexus injuries at $1.5 \mathrm{~T}$. Invest Radiol 48: 104-112.

4. Oberlin Cal, Béal D, Leechavengvongs S, Salon A, Dauge MC, et al. (1994) Nerve transfer to biceps muscle using a part of ulnar nerve for C5-C6 avulsion of the brachial plexus: anatomical study and report of four cases. J Hand Surg Am 19: 232-237.

5. Gu YD (2007) Contralateral C7 transfer over the last 20 years in China. Chin Med J (Engl) 120: 1123-1126.

6. Medical Research Council Scale (1976). Aids to the examination of the peripheral nervous system. Memorandum no 45, London, Her Majesty's Stationary Office.

7. Ferraresi S, Garozzo D, Buffatti P (2004) Reinnervation of the biceps in C5-7 brachial plexus avulsion injuries: results after distal bypass surgery. Neurosurg Focus 16: E6.

8. Midha R (1997) Epidemiology of brachial plexus injuries in a multitrauma population. Neurosurgery 40: 1182-1189

9. Malessy MJ, Hoffmann CF, Thomeer RT (1999) Initial report on the limited value of hypoglossal nerve transfer to treat brachial plexus root avulsions. $J$ Neurosurg 91: 601-604.

10. Ferraresi S, Garozzo D, Ravenni R, Dainese R, De Grandis D, et al. (2002) Hemihypoglossal nerve transfer in brachial plexus repair: technique and results. Neurosurgery 50: 332-335.

11. Midha R (2004) Nerve transfers for severe brachial plexus injuries: a review. Neurosurg Focus 16: E5.

12. Shin AY (2004) Nerve transfers for brachial plexus injuries. Oper Tech Orthop 14: 199-212.

13. El-Gammal TA, Fathi NA (2002) Outcomes of surgical treatment of brachia plexus injuries using nerve grafting and nerve transfers. J Reconstr Microsurg 18: 7-15.

14. Malessy M, van Duinen SG, Feirabend HK, Thomeer RT (1999) Correlation between histopathology of $\mathrm{C} 5$ and $\mathrm{C} 6$ nerve stumps and motor recovery following nerve grafting in brachia plexus repair. In: Brachial Plexus Surgery. Factors affecting functional recovery. J Neurosurg 636-644.

15. Bertelli JA, Ghizoni MF (2007) Transfer of the accessory nerve to the suprascapular nerve in brachial plexus reconstruction. J Hand Surg Am 32 989-998.

16. Luedemann W, Hamm M, Blomer U, Samii M, Tatagiba M, et al. (2002) Brachia plexus neurotization with donor phrenic nerves and its effect on pulmonary function. J Neurosurg 96: 523-526.

17. Hua XY, Liu B, Qiu YQ, Tang WJ, Xu WD, et al. (2013) Long-term ongoing cortical remodeling after contralateral C-7 nerve transfer. J Neurosurg 118: 725-729.
This article was originally published in a special issue, Neurorehabilitation \& Neural Repair handled by Editor(s). Dr. Hsinlin Thomas Cheng, University Of Michigan, USA 